\title{
Primary Antibiotic Resistance to Helicobacter pylori Strains Isolated From Children in Northern Iran: A Single Center Study
}

\author{
Shohreh Maleknejad, ${ }^{1, *}$ Ali Mojtahedi, ${ }^{1}$ Afshin Safaei-Asl, ${ }^{1}$ Zeinab Taghavi, and Ehsan \\ Kazemnejad $^{1}$
}

${ }^{1}$ Department of Pediatrics, 17th Shahrivar Hospital, Guilan University of Medical Sciences, Rasht, IR Iran

${ }^{*}$ Corresponding author: Shohreh Maleknejad, Department of Pediatrics, 17th Shahrivar Hospital, Guilan University of Medical Sciences, Rasht, IR Iran. Tel: +98-1313226101, Fax: +98-1313226101, E-mail: maleknejadshohreh@yahoo.com

Received 2015 June 3; Revised 2015 August 25; Accepted 2015 September 5

\begin{abstract}
Background: Initial resistance to antibiotics is the main reason for the failure of Helicobacter pylori (H. pylori) eradication in children. Objectives: As we commonly face high antibiotic resistance rates in children, we aimed to determine the susceptibility of $H$. pylori to common antibiotics.

Patients and Methods: In this cross-sectional in vitro study, 169 children younger than 14 years with clinical diagnosis of peptic ulcer underwent upper gastrointestinal endoscopy. Biopsy specimens from stomach and duodenum were cultured. In isolated colonies, tests of catalase, urease, and oxidase as well as gram staining were performed. After confirming the colonies as H. pylori, the antibiogram was obtained using disk diffusion method.

Results: Culture for $H$. pylori was positive in $12.3 \%$ of the specimens, urease test in $21.3 \%$, serological test in $18.9 \%$ and stool antigen test was positive in $21.9 \%$. We could show high specificity but moderate sensitivity of both histological and $H$. pylori stool antigen tests to detect H. pylori. The overall susceptibility to metronidazole was $42.9 \%$, amoxicillin $95.2 \%$, clarithromycin $85.7 \%$, furazolidone $61.9 \%$, azithromycin $81.0 \%$, and tetracycline $76.2 \%$ with the highest resistance to metronidazole and the lowest to clarithromycin.

Conclusions: In our region, there is high resistance of $H$. pylori to some antibiotics including metronidazole and furazolidone among affected children. To reduce the prevalence of this antibiotic resistance, more controlled use of antibiotics should be considered in children.

Keywords: Antibiotics, Culture, Resistance, Children, Helicobacter pylori
\end{abstract}

\section{Background}

Helicobacter pylori is a limpy gram-negative bacillus with positive catalase, oxidase and urease tests. $H$. pylori is found in half of the world population (1), its prevalence is highly variable in relation to geography, ethnicity, age, and socioeconomic factors, being high in developing countries and lower in the developed world $(2,3)$.

Eradication of $H$. pylori infection has currently become an important concern because it can cause many gastroduodenal disorders. During the last years, widespread use of antibiotics for the treatment of other diseases including parasitic infections, respiratory or intestinal disorders has led to increased antibiotic resistance against this bacterium. The resistance is substantially different among various communities (4-6).

Bacterial culture is the most common and specific invasive diagnostic method to detect the antimicrobial susceptibility of $H$. pylori, although its sensitivity can be largely variable (60\% - 90\%) mainly due to several methodological factors such as biopsy site, transport medium, time elapsed from sampling to processing, culture me- dium, and incubation conditions. Upper gastrointestinal endoscopy to obtain biopsy samples is the most reliable method to confirm $H$. pylori infection.

The most common antibiotics used in $H$. pylori treatment in children are amoxicillin, clarithromycin, and metronidazole or imidazole. The results of assessing antibiotic resistance in pediatric patients around the world can help select effective drugs for the prevention of treatment failure.

\section{Objectives}

As we commonly face high antibiotic resistance in children, so we aimed to determine the susceptibility of $H$. pylori to common antibiotics isolated from children in our region.

\section{Patients and Methods}

This was a cross-sectional in vitro study. 169 children (86 girls and 83 boys; age range 1.1 to 15 [mean $7.30 \pm 3.12$ ] years) clinically diagnosed as peptic ulcer disease underwent

Copyright (C) 2015, Growth \& Development Research Center. This is an open-access article distributed under the terms of the Creative Commons Attribution-NonCommercial 4.0 International License (http://creativecommons.org/licenses/by-nc/4.0/) which permits copy and redistribute the material just in noncommercial usages, provided the original work is properly cited. 
endoscopy. Three biopsies per patient from stomach and duodenum were taken for rapid urease test, pathological evaluation and culture. The samples were transferred to the microbiology laboratory of medical faculty within 3 hours. The study was undertaken in 17 Shahrivar pediatric hospital in Rasht, Guilan province, northern Iran, from March 2012 to March 2014. Exclusion criteria included treatment with proton pump inhibitor, trauma, burns and other stress situations as well as use of antibiotics in the past 2 weeks and nonsteroidal anti-inflammatory medication.

Biopsy specimens were cultured in Colombia agar medium containing sheep blood, fetal bovine serum FCS and antibiotic supplements. These were incubated 3 to 5 days in microaerophilic condition containing $\mathrm{CO}_{2} 5 \%$ at $37^{\circ} \mathrm{C}$. When colonies were observable, the tests for catalase, urease, oxidase and gram staining were performed. After confirming the colonies as H. pylori, the antiprogram was obtained using disk diffusion method on Mueller Hinton agar medium containing metronidazole, amoxicillin, clarithromycin, furazolidone, azithromycin, and tetracycline. The results were interpreted based on the presence or absence of inhibition zones and inhibition zone diameter created in the plate and were finally described as susceptible or resistant by comparison with the standard tables for interpretation of inhibition zones.

Results were presented as mean \pm standard deviation or median (1st, 3rd quartiles) for quantitative variables and were summarized by frequency (percentage) for categorical variables. Continuous variables were compared using $\mathrm{t}$ test or Mann-Whitney $\mathrm{U}$ test whenever the data did not appear to have normal distribution or when the assumption of equal variances was violated across the study groups. Categorical variables were, on the other hand, compared using Chi-square test. For the statistical analysis, the statistical software SPSS version 20.0 for windows (SPSS Inc., Chicago, IL) was used. P values of 0.05 or less were considered statistically significant.

\section{Results}

Urease test was positive in $21.3 \%$ of the samples and serology test in $18.9 \%$ of the studied children. Both culture and $H$. pylori stool antigen (HPSA) test were positive in $24.3 \%$ with a sensitivity of $42.9 \%$ and specificity of $81.1 \%$ for HPSA test to detect $H$. pylori infection.

Of 169 culture specimens, 21 (12.3\%) were positive for $H$. pylori, $19.1 \%$ of which were sensitive and $9.5 \%$ resistant to all studied antibiotics. The highest and lowest resistance rates were related to metronidazole (57.1\%) and clarithromycin (4.8\%) (Figure 1). As shown in Figure 2, comparing resistance rates to various types of antibiotics between male and female subjects none of them showed a difference. Also, regarding H. pylori resistance to different types of antibiotics in different age subgroups (Table 1), except for amoxicillin that had a higher susceptibility rate among younger children, the susceptibility to other antibiotics was similar between younger and older children.

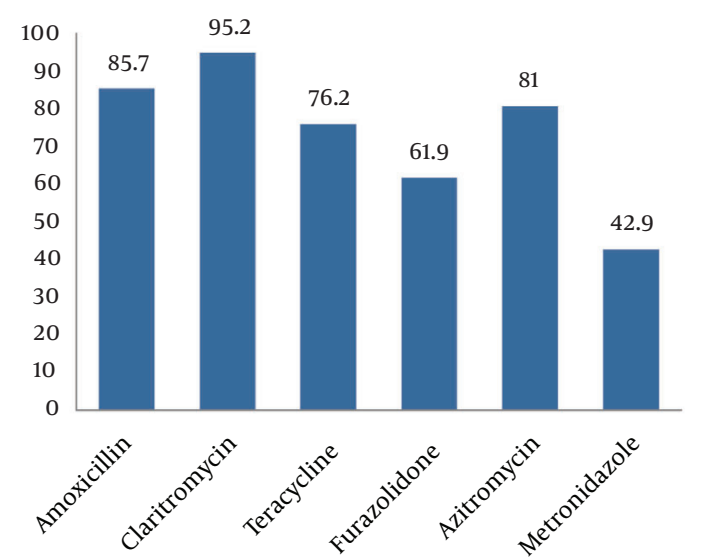

Figure 1. Helicobacter pylori Susceptibility to Antibiotics

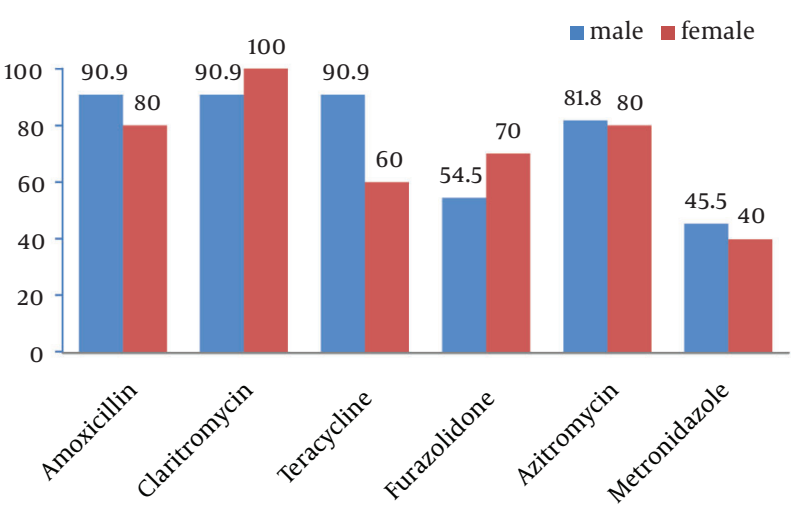

Figure 2. Helicobacter pylori Susceptibility to Different Antibiotics in Male and Female Subjects

Table 1. Helicobacter pylori Susceptibility to Different Antibiotics According to Mean Age

\begin{tabular}{lccc}
\hline Antibiotic & Susceptibility & Resistance & PValue \\
\hline Amoxicillin & $8.21 \pm 2.57$ & $12.33 \pm 1.89$ & 0.016 \\
Claritromycin & $8.55 \pm 2.68$ & $13.80 \pm 1.00$ & 0.071 \\
Tetracycline & $8.37 \pm 2.87$ & $10.18 \pm 2.61$ & 0.224 \\
Furazolidone & $8.28 \pm 3.03$ & $9.64 \pm 2.50$ & 0.303 \\
Azitromycin & $8.44 \pm 2.80$ & $10.35 \pm 2.92$ & 0.237 \\
Metronidazole & $9.52 \pm 2.78$ & $8.26 \pm 2.91$ & 0.328 \\
\hline
\end{tabular}

\section{Discussion}

H. pylori infection has important implications for health and remains a major healthcare burden with persistently high prevalence, especially in less-developed countries. The eradication of $H$. pylori infection represents an enormous challenge in gastroenterology. However, $H$. pylori resistance to antibiotics is the major factor affecting the efficacy of current therapeutic regimens (1-3).

Considering that this organism lives in an environment 
not easily accessible to many drugs, the increasing antibiotic resistance can be a difficult task. To know the local prevalence of antibiotic resistance is important also for choosing the better therapy mainly if the antimicrobial susceptibility does not develop from the culture.

In our study, $12.3 \%$ of cultures, $21.3 \%$ of urease tests, $18.9 \%$ serological tests and $21.9 \%$ of stool antigen tests of the specimens were positive. Overall we could show high specificity but moderate sensitivity of both histological and HPSA tests to detect H. pylori.

Since patients were referred to an exclusive pediatric gastroenterology center within province, it is possible that the prevalence of $H$. pylori infection was higher than that in the general population. The antimicrobial resistance pattern of $H$. pylori in this study was consistent with similar studies from Asia, Middle East and developing countries (4-6).

Level of resistance differs from country to country. Siavoshi et al. (7) showed similar rates of resistance to metronidazole, clarithromycin, amoxicillin, tetracycline and furazolidone of $H$. pylori isolates from adults and children (7).

In the present study, there was no direct association between sex and age with metronidazole, clarithromycin, amoxicillin, tetracycline, furazolidone, and clarithromycin resistance which is in agreement with some similar studies in Iran and other countries $(4,8,9)$. Unlike these results, Siavoshi et al. (7) in Tehran/Iran and Kato and coworkers (10) in Japan reported a statistically significant association between clarithromycin resistance and pediatric age group.

Also Koletzko et al. found that metronidazole resistance was more frequent in $\mathrm{H}$. pylori isolates from female than male patients where metronidazole was widely used to treat parasitic diseases and gynecological infections (11). Because of this, gender has been suggested to be a risk factor for resistance to metronidazole.

In our study, high resistance to antibiotics was found for metronidazole and low resistance for clarithromycin. In this study, the prevalence of resistance to metronidazole with $57.1 \%$ was high. This has been reported from Iran and some other Asian countries too (8-10, 12-15), being related to the widespread use of this antibiotic in these countries. Overuse of antibiotics and limited options has led to increased resistance of the microorganism. The rate of resistance to metronidazole or other antimicrobial agents fluctuates in the world and it depends on poor socio-economics, life condition, geographical area, drug doses, and period of treatment and excessive administration of antibiotics due to other infections.

In our study the primary resistance rate of $H$. pylori isolates to the clarithromycin in children was $4.8 \%$. In contrast to bacterial resistance to metronidazole, the prevalence of $H$. pylori resistance to clarithromycin is much lower. In developed countries, approximately $10 \%$ of the $H$. pylori strains are clarithromycin-resistant, but in developing countries, this resistance rate is higher ranging from $25 \%$ to $50 \%$. The rate of resistance to clarithromycin also fluctuates in different parts of Iran. Six studies in different regions by Siavoshi et al. (7), Milani et al. (12), Fallahi and Maleknejad (14), Falsafi et al. (16), Tomatari et al. (17) , Mohammadi et al. (18), and Naserpour Farivar et al. (19) showed the rate of resistance to clarithromycin as $14.5 \%, 14 \%, 4.16 \%, 14.5 \% 23 \%, 21 \%, 16.7 \%, 12 \%$, respectively. This reflects the importance of diverse geographical areas. Our results were compatible with resembling studies reported from the Middle Eastern countries Saudi Arabia (4\%), Kuwait (0\%) and Egypt (4\%) $(4,20)$. Based on these results, resistance rate of $H$. pylori strains to clarithromycin in Iran is lower than in other developing countries, and is higher than in Middle Eastern and developed countries. Oleastro et al. study revealed no changes in clarithromycin nor in metronidazole resistance over a 10- year period in Portuguese children (21). Kalach and coworkers showed a decrease in metronidazole resistant strains but they did not observe changes in clarithromycin resistance in French children over 11 years (22). The essential risk factor for clarithromycin resistance is previous consumption of macrolides, and higher resistance in children is caused by increased prescription of these drugs in children notably during the last decade especially for respiratory tract infections.

In our research, amoxicillin resistance in children was $14,3 \%$. In all of the surveys reported resistance to amoxicillin is rare, as well as in recent studies from Europe (3), Portugal (21), Austria (23), Vietnam (24), Italy (25), and Mexico (26).

The findings of several studies revealed that the highest (59\%) and lowest ( $0 \%$ ) rate of resistance to amoxicillin in Iran were reported from Tabriz and Tehran, respectively $(7,13)$.

It seems that resistance rate to amoxicillin in Iran is higher than in other countries especially in Europe, United States, Canada, which reflects the effect of its overuse in our country, especially in children. This is an alarming condition as this drug is the most effective antibiotic for eradicating the organism.

Our study, as well as almost all studies in our region provided evidence for high resistance to widely used drugs including metronidazole and high susceptibility to low consumed drugs including clarithromycin and furazolidone. Also, despite low consumption of tetracycline, resistance to this antibiotic is reported to be considerably high. Despite contraindications for the use of tetracycline in children younger than 9 years, the resistance rate to this antibiotic in our study was considerably high $(23.8 \%)$ probably due to increased resistant strains in infected adults in this region.

Moreover, because of high resistance to azithromycin, this drug should not be considered as a choice for eradicating $H$. pylori. Furazolidone and metronidazole resistance in our study was higher because of the high prevalence of parasitic infestations in northern parts of the country, and more uncritical use of antibiotics in these 
areas. Given the importance of eradication of H.pylori infection, appropriate antibiotics should be used with less resistance. Furthermore, to reduce the prevalence of antibiotic resistance, more critical use of relevant antibiotics should be considered in pediatric patients.

To achieve a successful eradication of $H$. pylori, sufficient knowledge of the germ susceptibility and the regional distribution of resistant strains is essential. It must also be kept in mind that the susceptibility to drugs may alter in the population. So, to prevent antibiotic resistance and to determine the most effective drugs, continuous surveillance is needed.

\section{Acknowledgments}

This study was supported by Guilan University of Medical Sciences, Rasht.

\section{References}

1. Marcdante K, Kliegman RM, Behrman RE, Jenson HB. Nelson essentials of pediatrics. Elsevier Health Sciences; 2010.

2. Sykora J, Rowland M. Helicobacter pylori in pediatrics. Helicobacter. 2011;16 Suppl 1:59-64. doi: 10.1111/j.1523-5378.2011.00882.x. [PubMed: 21896087]

3. Calvet X, Ramirez Lazaro MJ, Lehours P, Megraud F. Diagnosis and epidemiology of Helicobacter pylori infection. Helicobacter 2013;18 Suppl 1:5-11. doi: 10.1111/hel.12071. [PubMed: 24011238]

4. Megraud F. H pylori antibiotic resistance: prevalence, importance, and advances in testing. Gut. 2004;53(9):1374-84. doi 10.1136/gut.2003.022111. [PubMed:15306603]

5. European Helicobacter Pylori Study Group Current European concepts in the management of Helicobacter pylori infection. The Maastricht Consensus Report. European Helicobacter Pylori Study Group. Gut. 1997;41(1):8-13. doi: 10.1136/gut.41.1.8. [PubMed: 9274464]

6. Jason LA, Pokorny SB, Adams M, Nihls A, Kim HY, Hunt Y. Cracking Down On Youth Tobacco May Influence Drug Use. J Community Psychol. 2010;38(1):1-15. doi: 10.1002/jcop.20347. [PubMed: 21850151]

7. Siavoshi F, Safari F, Doratotaj D, Khatami GR, Fallahi GH, Mirnaseri MM. Antimicrobial resistance of Helicobacter pylori isolates from Iranian adults and children. Arch Iran Med. 2006;9(4):30814. [PubMed:17061600]

8. Eyvazi S, Hakemi-Vala M. A Review Article on Helicobacter pylori Antibiotic Resistance Profile in Iran. Int J Trop Dis Health. 2015;10(1):1-12. doi:10.9734/ijtdh/2015/18719.

9. Khademi F, Poursina F, Hosseini E, Akbari M, Safaei HG. Helicobacter pylori in Iran: A systematic review on the antibiotic resistance. Iran J Basic Med Sci. 2015;18(1):2-7. [PubMed: 25810869]

10. Kato S, Fujimura S, Udagawa H, Shimizu T, Maisawa S, Ozawa $\mathrm{K}$, et al. Antibiotic resistance of Helicobacter pylori strains in Japanese children. J Clin Microbiol. 2002;40(2):649-53. [PubMed 11825987]

11. Koletzko S, Richy F, Bontems P, Crone J, Kalach N, Monteiro ML, et al. Prospective multicentre study on antibiotic resistance of Helicobacter pylori strains obtained from children living in Europe Gut. 2006;55(12):1711-6. doi: 10.1136/gut.2006.091272. [PubMed: 16603633]

12. Milani M, Ghotaslou R, Akhi MT, Nahaei MR, Hasani A, Somi MH, et al. The status of antimicrobial resistance of Helicobacter pylori in Eastern Azerbaijan, Iran: comparative study according to demographics. J Infect Chemother. 2012;18(6):848-52. doi: 10.1007| s10156-012-0425-4. [PubMed: 22581031]

13. Rafeey M, Ghotaslou R, Nikvash S, Hafez AA. Primary resistance in Helicobacter pylori isolated in children from Iran. $J$ Infect Chemother. 2007;13(5):291-5. doi: 10.1007/s10156-007-0543-6. [PubMed:17982716]

14. Fallahi GH, Maleknejad S. Helicobacter pylori culture and antimicrobial resistance in Iran. Indian J Pediatr. 2007;74(2):127-30. doi:10.1007/s12098-007-0003-4. [PubMed:17337822]

15. Alborzi A, Soltani J, Pourabbas B, Oboodi B, Haghighat M, Hayati $\mathrm{M}$, et al. Prevalence of Helicobacter pylori infection in children (south of Iran). Diagn Microbiol Infect Dis. 2006;54(4):259-61. doi: 10.1016/j.diagmicrobio.2005.10.012. [PubMed:16466888]

16. Falsafi T, Mobasheri F, Nariman F, Najafi M. Susceptibilities to different antibiotics of Helicobacter pylori strains isolated from patients at the pediatric medical center of Tehran, Iran.J Clin Microbiol. 2004;42(1):387-9. [PubMed:14715786]

17. Haghi Tomatari F, Mohabbati Mobarez A, Amini M, Hosseini D, Abadi ATB. Helicobacter pylori resistance to metronidazole and clarithromycin in dyspeptic patients in Iran. Iran Red Crescent Med J. 2010;12(4):409.

18. Mohammadi M, Doroud D, Mohajerani N, Massarrat S. Helicobacter pylori antibiotic resistance in Iran. World J Gastroenterol. 2005;11(38):6009-13. [PubMed:16273615]

19. Naserpour Farivar T, Najafipour R, Johari P. Prevalence of clarithromycin-resistant Helicobacter pylori in patients with chronic tonsillitis by allele-specific Scorpion real-time polymerase chain reaction assay. Laryngoscope. 2013;123(6):1478-82. doi: 10.1002/lary.23777. [PubMed: 23404672]

20. Sherif M, Mohran Z, Fathy H, Rockabrand DM, Rozmajzl PJ Frenck RW. Universal high-level primary metronidazole resistance in Helicobacter pylori isolated from children in Egypt. $J$ Clin Microbiol. 2004;42(10):4832-4. doi: 10.1128/JCM.42.10.48324834.2004. [PubMed:15472354]

21. Oleastro M, Cabral J, Ramalho PM, Lemos PS, Paixao E, Benoliel J, et al. Primary antibiotic resistance of Helicobacter pylori strains isolated from Portuguese children: a prospective multicentre study over a 10 year period. I Antimicrob Chemother. 2011;66(10):2308-11. doi:10.1093/jac/dkr293. [PubMed: 21764826]

22. Kalach N, Serhal L, Asmar E, Campeotto F, Bergeret M, Dehecq E, et al. Helicobacter pylori primary resistant strains over 11 years in French children. Diagn Microbiol Infect Dis. 2007;59(2):217-22. doi: 10.1016/j.diagmicrobio.2007.05.003. [PubMed:17662555]

23. Vecsei A, Kipet A, Innerhofer A, Graf U, Binder C, Gizci H, et al Time trends of Helicobacter pylori resistance to antibiotics in children living in Vienna, Austria. Helicobacter. 2010;15(3):214-20. doi: 10.1111/j.1523-5378.2010.00753.x. [PubMed: 20557363]

24. Nguyen TV, Bengtsson C, Yin L, Nguyen GK, Hoang TT, Phung DC, et al. Eradication of Helicobacter pylori in children in Vietnam in relation to antibiotic resistance. Helicobacter. 2012;17(4):319-25. doi:10.1111/j.1523-5378.2012.00950.x. [PubMed: 22759333]

25. Torres J, Camorlinga-Ponce M, Perez-Perez G, Madrazo-De la Garza A, Dehesa M, Gonzalez-Valencia G, et al. Increasing multidrug resistance in Helicobacter pylori strains isolated from children and adults in Mexico. J Clin Microbiol. 2001;39(7):2677-80. doi: 10.1128/JCM.39.7.2677-2680.2001. [PubMed: 11427594]

26. Manfredi M, Gismondi P, Maffini V, Bizzarri B, Fornaroli F, Madia C, et al. Primary Antimicrobial Susceptibility Changes in Children with Helicobacter pylori Infection over 13 Years in Northern Italy. Gastroenterol Res Pract. 2015;2015:717349. doi: 10.1155/2015/717349. [PubMed: 26064096] 